\title{
Germaphobia! Does Our Relationship With and Knowledge of Biodiversity Affect Our Attitudes Toward Microbes?
}

\author{
Jake M. Robinson ${ }^{1,2,3 *}$, Ross Cameron ${ }^{1}$ and Anna Jorgensen ${ }^{1}$ \\ ${ }^{1}$ Department of Landscape Architecture, The University of Sheffield, Sheffield, United Kingdom, ${ }^{2}$ inVIVO Planetary Health, \\ Worldwide Universities Network, Jersey City, NJ, United States, ${ }^{3}$ The Healthy Urban Microbiome Initiative (HUMI), Adelaide, \\ SA, Australia
}

OPEN ACCESS

Edited by:

Silvia Collado,

University of Zaragoza, Spain

Reviewed by:

Victor Corral-Verdugo, University of Sonora, Mexico

Stepan Vesely,

Norwegian University of Science and Technology, Norway

*Correspondence:

Jake M. Robinson

jmrobinson3@sheffield.ac.uk

Specialty section:

This article was submitted to

Environmental Psychology, a section of the journal

Frontiers in Psychology

Received: 10 March 2021

Accepted: 01 June 2021

Published: 30 June 2021

Citation:

Robinson JM, Cameron $R$ and Jorgensen A (2021) Germaphobia!

Does Our Relationship With and Knowledge of Biodiversity Affect

Our Attitudes Toward Microbes?

Front. Psychol. 12:678752.

doi: 10.3389/fpsyg.2021.678752
Germaphobia - a pathological aversion to microorganisms - could be contributing to an explosion in human immune-related disorders via mass sterilization of surfaces and reduced exposure to biodiversity. Loss of biodiversity and people's weaker connection to nature, along with poor microbial literacy may be augmenting the negative consequences of germaphobia on ecosystem health. In this study, we created an online questionnaire to acquire data on attitudes toward, and knowledge of microbes. We collected data on nature connectedness and interactions with nature and explored the relationships between these variables. Although the study had an international reach $(n=1,184)$, the majority of responses came from England, United Kingdom ( $n=993)$. We found a significant association between attitudes toward microbes and both duration and frequency of visits to natural environments. A higher frequency of visits to nature per week, and a longer duration spent in nature per visit, was significantly associated with positive attitudes toward microbes. We found no association between nature connectedness and attitudes toward microbes. We found a significant relationship between knowledge of "lesser known" microbial groups (e.g., identifying that fungi, algae, protozoa, and archaea are microbes) and positive attitudes toward microbes. However, we also found that people who identified viruses as being microbes expressed less positive views of microbes overall-this could potentially be attributed to a "COVID19 effect." Our results suggest that basic microbial literacy and nature engagement may be important in reducing/preventing germaphobia-associated attitudes. The results also suggest that a virus-centric phenomenon (e.g., COVID-19) could increase broader germaphobia-associated attitudes. As the rise of immune-related disorders and mental health conditions have been linked to germaphobia, reduced biodiversity, and nontargeted sterilization, our findings point to a feasible strategy to potentially help ameliorate these negative consequences. Further research is needed, but greater emphasis on microbial literacy and promoting time spent in nature could potentially be useful in promoting resilience in human health and more positive/constructive attitudes toward the foundations of our ecosystems - the microorganisms.

Keywords: microbiome, microorganisms, COVID-19, germaphobia, mysophobia, nature connectedness, nature relatedness 


\section{INTRODUCTION}

Germaphobia-also known as "mysophobia" - is the pathological fear of, and aversion to dirt and microorganisms (henceforth referred to as "microbes") (Zemke et al., 2015). The rise of germaphobia has likely been influenced by decades of advertising campaigns creating negative perceptions of microbes, and falsely prompting mass (non-targeted) sterilization of surfaces to achieve "safe" human environments (Timmis et al., 2019). Symptoms of germaphobia include avoiding certain "dirty" environments (e.g., soil) due to perceived to fear of microbial exposure, excessively washing hands, over-use of sanitizers and antibiotics (Qadir and Yameen, 2019). However, far less than $1 \%$ of the microbes on the planet are human pathogens (Zobell and Rittenberg, 2011; Balloux and van Dorp, 2017). Moreover, germaphobia may have contributed to the current explosion in human immune-related disorders (such as diabetes, asthma, and inflammatory bowel disease) (Jun et al., 2018; Timmis et al., 2019). This is thought to be attributed to the notion that exposure to environmental microbiomes-the diverse network of microbes in a given environment-plays an important role in human health (Rook et al., 2003; Dannemiller et al., 2014; Stein et al., 2016; Arleevskaya et al., 2019; Liddicoat et al., 2019; Selway et al., 2020). Indeed, from a young age, exposure to a diverse range of environmental microbes is considered to be essential for the assembly of our microbiome and the training and regulation of our immune systems (Flies et al., 2020; Renz and Skevaki, 2020; Roslund et al., 2020). A stable and functional human microbiome is colonized following birth. Firstly by the mother's skin and breast milk, and later supplemented from visitors, pets, biodiverse environments, and a "normal dirty" (not overly cleaned) home environment (DeWeerdt, 2018). Germaphobia and associated overly-clean disposition (whilst recognizing targeted hygiene is essential) could conceivably inhibit all of these activities (e.g., avoiding playing in soil or staying away from animals), and if the microbiome assembly process is derailed, the negative health consequences such as immune dysfunction, could be long-term (Gensollen et al., 2016; Renz and Skevaki, 2020). In relation to the current COVID-19 pandemic - a situation that could conceivably increase germaphobia - in addition to being hygienic, we need to promote the concept that the majority of microbes are in fact innocuous and/or beneficial to human health via immunoregulation and other functional roles (Rook, 2013). Indeed, through the modulation of host immune responses, the gut microbiome may even have a direct role in regulating COVID-19 severity (Yeoh et al., 2021).

Microbial communities and their interactions also play essential roles in carbon and nutrient cycling, climate regulation, animal and plant health, and global food security (Cavicchioli et al., 2019; Li et al., 2020; Trivedi et al., 2020). Therefore, microbial biodiversity is of vital importance for the ability of ecosystems to simultaneously provide multiple ecosystem services (Guerra et al., 2020). Consequently, ongoing degradation of microbial communities likely poses an important threat to global macro-level biodiversity and to human societies across the planet (Cavicchioli et al., 2019). Loss of biodiversity and our affective, cognitive and experiential connection with the natural world (also known as "nature connectedness"), along with poor microbial literacy (such as awareness of the different types of microbes and their importance) and germaphobia, may be detrimental to ecosystem health (Cavicchioli et al., 2019; Robinson and Breed, 2020). Studies have suggested that environmental knowledge (particularly of macro-ecological features) can play a role in fostering pro-ecological attitudes and behaviors (Sat Gungor et al., 2018; Choe J. H. et al., 2020), while other suggest knowledge is not an important factor (Qomariah and Prabawani, 2020). A recent study investigated the factors that account for pro-ecological behaviors, and found that nature connectedness, nature experiences (time spent in nature and nature engagement) and nature-based knowledge and attitudes explained $70 \%$ of the variation in people's actions for nature (Richardson et al., 2020). Other studies have shown that connectedness to nature and frequency of visits to nature is linked to pro-ecological behaviors (Collado et al., 2015; DuronRamos et al., 2020). Recent work suggested that outdoor nature experiences can help overcome fears of "creepy crawlies" such as insects and snakes and can help develop respectful and positive attitudes toward nature (Hosaka et al., 2017; Chawla, 2020).

Is our diminishing connection with (the rest of) the natural world helping to drive germaphobia-associated attitudes (which may subsequently affect behaviors)? To our knowledge, no studies have investigated the relationship between nature engagement (duration and frequency in nature), nature connectedness and attitudes toward the invisible constituents of nature (i.e., microorganisms). Furthermore, no studies have explored whether there is a relationship between basic knowledge of microorganisms and attitudes toward microorganisms.

In this study, we used an online questionnaire to acquire data on attitudes toward microbes. We collected data on respondents' nature engagement (including typical duration and frequency of visits to nature), and data on nature connectedness using the Nature Relatedness 6 Scale-a validated psychological instrument (Nisbet and Zelenski, 2013). To gauge respondents' basic knowledge of microbes, we asked them to select all of the organisms (from a list) that they considered to be microbes. The relationships between these variables (i.e., between nature connectedness, nature engagement and attitudes toward microbes; and between basic microbial literacy and attitudes toward microbes) were then assessed using a range of statistical methods including logistic regression models, Mann-Whitney $U$ tests, and 2-sample tests for equality of proportions with continuity correction in R.

The primary objectives of this study were to: (a) assess whether people's patterns of exposure to nature associated with their attitudes toward microbes (i.e., a positive or negative view); (b) assess whether people's level of subjective connectedness to nature associated with their attitudes toward microbes; and, (c) investigate whether basic knowledge of microbial groups (e.g., identifying that fungi, algae, protozoa, and archaea are also microbes) associated with attitudes toward microbes.

Gaining a better understanding of the factors that may aid in reducing/preventing germaphobia-associated attitudes (e.g., negative attitudes that may influence subsequent behaviors) could help to inform environmental and public health policy. For 
example, improving microbial literacy and promoting campaigns that seek to reconnect humans with the wider biotic community could potentially bring value to both human and environmental health. Microbes are the foundations of our ecosystems and are essential to the survival of all life on Earth (Cavicchioli et al., 2019). Targeted hygiene approaches and continued efforts to control infectious diseases are undoubtedly vital. However, germaphobia (and associated actions such as soil/nature avoidance, and mass sterilization of the environment) only serves to inhibit a more nuanced awareness of, and mutuallyadvantageous relationship with these diverse, underappreciated, and indispensable lifeforms.

\section{MATERIALS AND METHODS}

\section{Online Questionnaire}

We produced a research questionnaire using the Smart Survey online software (Smart Survey, 2020). The questionnaire included 21 multi-format questions (Supplementary Appendix A). The questions were devised to gather data on respondents based on four variables: (1) nature engagement (via determining frequency and duration in nature); (2) nature connectedness; (3) attitudes toward microbes; and, (4) basic knowledge of microbes. The online survey was active between April and July 2020.

\section{Nature Engagement}

As the study was conducted during the COVID-19 pandemic, we asked participants to provide answers by referring to their typical patterns of visiting nature before the pandemic. For example, the following questions were asked: "how many times would you visit any natural environments (e.g., parks, woodlands, and the beach) in a typical week before the COVID-19 pandemic?"; and "Approximately how long would you spend in any natural environment per visit before the COVID-19 pandemic?" For this study "natural environments" and/or "nature" were considered to be less anthropogenic/builtup environments, typically containing a large proportion of vegetation and wildlife such as woodlands, parks, and meadows.

\section{Nature Connectedness}

We asked participants to answer questions regarding how emotionally and cognitively connected they felt to nature using the Nature Relatedness Scale (NR-6) (Nisbet and Zelenski, 2013; Kettner et al., 2019). The NR-6 comprises six questions, and answers are recorded using a 1-5 Likert scale. Examples of questions include "My relationship to nature is an important part of who I am," "My ideal vacation spot would be a remote, wilderness area," and "I feel very connected to all living things and the earth." Items were averaged, and higher scores indicated stronger subjective connectedness to nature. This validated instrument has been used in several previous environmental psychology studies (Nisbet and Zelenski, 2013; Obery and Bangert, 2017; Whitburn et al., 2020). We also asked several pilottested questions regarding typical exposure to nature such as duration and frequency of visits to natural environments.

\section{Attitudes Toward Microbes}

To acquire data on respondents' attitudes toward microbes, we devised a pilot-tested word-association measure using three categories: positive association, neutral association, and negative association. To reduce potential bias, the categories were not revealed to the respondents and each category contained five randomly-ordered words, displayed as one amalgamated list (Supplementary Appendix A). In the positive category, respondents could choose from words such as "essential" and/or "beneficial." In the neutral category respondents could choose from words such as "nature" and/or "mobile." In the negative category respondents could choose from words such as "disease" and/or "nuisance." Respondents were asked to select a total of three words that best reflected their view of microbes. We also used the questions "do you consider microbes to be good?; bad?; some are good, some are bad?; or, neither are good or bad?," the resulting positive and negative categories were used in the models to explore the influence of nature connectedness. To gauge respondents' basic knowledge of microbes, we asked them to select all of the organisms that they considered to be microbes. The list included bacteria, viruses, fungi, algae, protozoa, and archaea. Due to the current COVID-19 pandemic, which is of viral origin, we separated out viruses in some of the analyses in case they affected people's overall perception of microbes.

\section{Demographic Data, Distribution, Exclusion, and Ethics}

We also acquired key demographic information including postal code, deprivation (based on the Index of Multiple Deprivation, which takes into account socioeconomic, occupational, housing, and environmental factors to estimate deprivation), age, gender, highest level of education, and occupation. The questionnaire, along with a detailed participant information sheet and consent form was distributed across the world via a secure weblink. We used several non-random sampling methods to reach respondents including: social media posting, emailing volunteer groups, and carrying out an online search of publicly available community group directories. The only exclusion criterion for the study was: people under 18 years of age. The questionnaire was ethically reviewed by the internal review committee in the Department of Landscape Architecture at the University of Sheffield (the authors' academic institution).

\section{Statistical Analysis}

To test the hypothesis that nature engagement i.e., duration and frequency of visits to nature, may positively influence a person's attitudes toward microbes, we acquired a score from the wordassociation output by summing the positive, neutral and negative values given by each respondent-this was used as a proxy to indicate positive vs. negative attitude toward microbes. We then assigned the positive and negative scores into two groups and compared the mean duration and frequency of visits to nature of each group using the two-sample Mann-Whitney $U$ test with continuity correction in $\mathrm{R}$.

To test the hypothesis that nature connectedness influences people's attitudes toward microbes, we built logistic regression models. For these models, an odds ratio (OR) of 1 or above 
equated to the predictor variable (nature connectedness score) increasing the odds of a positive attitude toward microbes. An $\mathrm{OR}<1$ equated to the predictor variable decreasing the odds of a positive attitude toward microbes. Answers from the question "do you consider microbes to be good" were coded into a "positive" category, and "do you consider microbes to be bad" were coded into a "negative" category, and these were then used in the regression models as binary dependent variables. We adjusted for several covariates including age, gender, deprivation, and level of education.

To test the hypothesis that basic knowledge of microbes influences people's attitudes toward microbes, we assessed proportional differences between groups, in which respondents either did or did not identify different microbial groups (i.e., bacteria, viruses, fungi, algae, protozoa, and archaea) and their respective word-association scores (summing the negative, neutral and positive scores as a proxy to indicate a positive or negative attitude as a variable) using the 2-sample tests for equality of proportions with continuity correction in R. For example, three positive words = net positive score; two positive words and one negative or neutral = net positive score, and the reverse formula was used to acquire a net negative score.

\section{RESULTS}

A total of $n=1,184$ respondents completed the questionnaire. A broad distribution of responses from across the world was acquired (Figure 1A); however, the main cluster $(n=993)$ was from England, United Kingdom (Figure 1B).

Respondents who identified as being female ( $n=851$ or $72 \%)$ outnumbered those who identified as being male $(n=331$ or $28 \%)$, trans woman $(n=1$ or $0.1 \%)$, and non-binary $(n=1$ or $0.1 \%)$. There was also a skew toward respondents with a higher level of education ( $n=847$ or $72 \%$ with $\geq$ undergraduate degree). In terms of age, the distribution either side of the median was similar ( $n=624$ or $53 \%$ were $\geq 55$ years old; and $n=560$ or $47 \%$ were $\leq 54$ years old).

\section{Nature Engagement, and Attitudes Toward Microbes}

Our results show that respondents with a net positive wordassociation score for microbes (i.e., those who viewed microbes more positively) spent significantly more time per visit ( $\mathrm{x}=87 \mathrm{~min}$ ) to natural environments such as woodlands, parks, and meadows compared to respondents with a net negative word-association score for microbes ( $\mathrm{x}=70 \mathrm{~min})(\mathrm{W}=3,995$, $p \leq 0.01$ ) (Figure 2).

Our results also show that respondents with a net positive word-association score for microbes visited natural environments such as woodlands, parks, and meadows significantly more often ( $\stackrel{\circ}{\mathrm{x}}=4.2$ visits in a given week) compared to respondents with a net negative word-association score for microbes $(\stackrel{\circ}{\mathrm{x}}=3.8$ visits in a given week) $(W=3,935$, $p \leq 0.01$ ) (Figure 3).

\section{Nature Connectedness and Attitudes Toward Microbes}

We found no association between nature connectedness (measured using the NR-6 Scale) and attitudes toward viruses (OR: $0.99(0.95,1.02) p=0.54)$ or all other microbes (OR: 1.01 $(0.89,1.16) p=0.86)$ (Table 1).

\section{Basic Microbial Literacy and Attitudes Toward Microbes}

Mean positive scores (derived from word-association) toward all microbes were significantly higher for those who correctly identified that fungi $\left(X^{2}=42.5, \mathrm{df}=1, p \leq 0.01\right)$ archaea $\left(X^{2}=52\right.$, $\mathrm{df}=1, p \leq 0.01)$ micro-algae $\left(X^{2}=30\right.$, $\left.\mathrm{df}=1, p \leq 0.01\right)$ and protozoa $\left(X^{2}=51\right.$, df $\left.=1, p \leq 0.01\right)$ were microbes compared to those who did not identify these groups as being microbes. Mean positive scores toward all microbes were significantly lower for those who identified that viruses were microbes compared to those who did not identify viruses as being microbes $\left(X^{2}=30.7\right.$, $\mathrm{df}=1, p \leq 0.01$ ). There were no significant differences in scores between respondents who correctly identified bacteria as being microbes $(n=1124)$ compared to those who did not $(n=60)$ $\left(X^{2}=<0.01, \mathrm{df}=1, p=1.0\right)$ (Figure 4).

\section{DISCUSSION}

Our study shows a significant positive relationship between nature engagement (a respondent's duration and frequency in nature) and the respondents' attitudes toward microbes. However, we found no association between nature connectedness (a person's affective, cognitive and experiential connection with the natural world) (Cheung et al., 2020; Choe E. Y. et al., 2020) and attitudes toward microbes. Importantly, we found a significant relationship between knowledge of "lesser known" microbial groups (e.g., identifying that fungi, algae, protozoa, and archaea are microbes) and positive attitudes toward microbes. This study suggests that nature engagement and basic microbial literacy may be important in improving positive attitudes toward microbes. Further confirmatory research is required, with a focus on whether these potential changes to attitudes translate to changes in germaphobia-associated behaviors.

As mentioned, nature engagement significantly associated with positive attitudes toward microbes. This finding supports our first hypothesis, and is corroborated by other (nonmicrobiological) work that suggests nature engagement may reduce fears of "creepy crawlies" and help foster respectful and positive attitudes toward nature (Hosaka et al., 2017; Chawla, 2020). It is important to note that the directionality of the relationship is unknown (i.e., whether spending more time in nature helps to establish more positive attitudes toward microbes, or whether other factors related to more positive attitudes increase the likelihood of spending more time in nature). Conceivably, being less averse to microbes could increase one's desire to spend time in environments with natural features such as plants and soil-key sources of dense microbial communities 


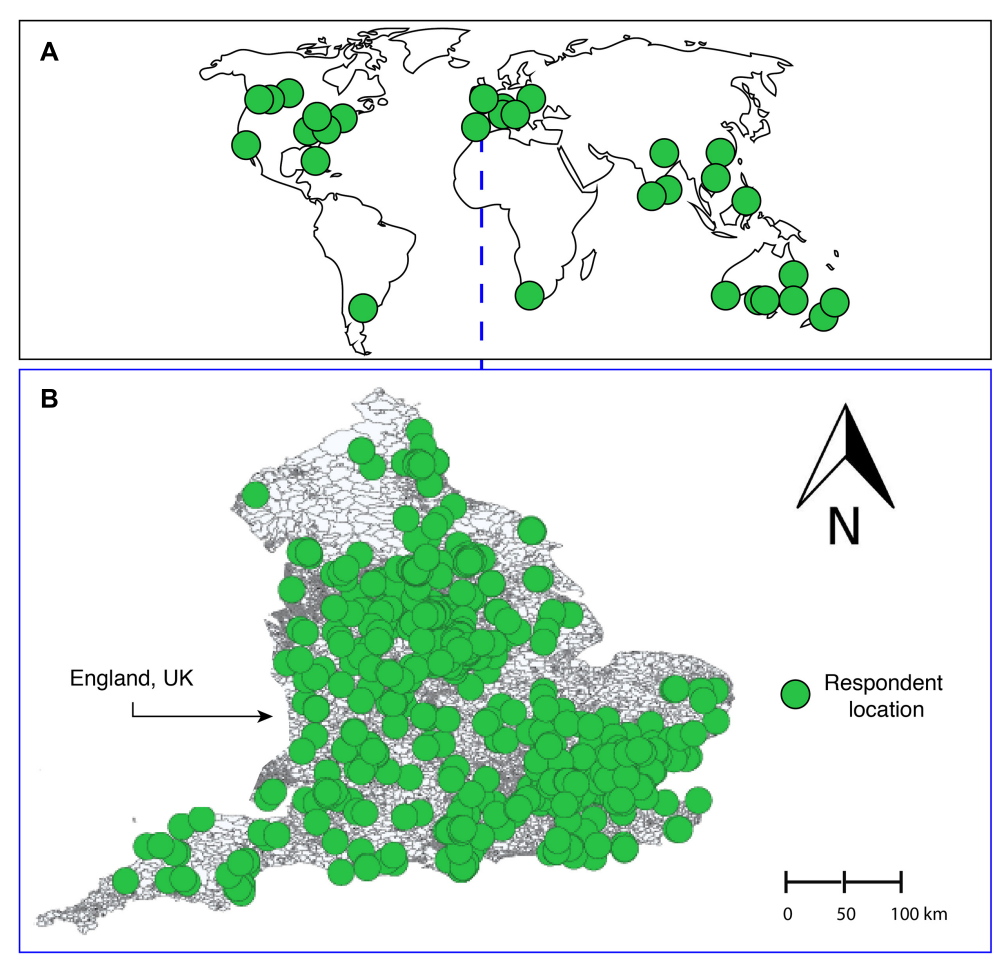

FIGURE 1 | Distribution of respondents, whereby panel (A) shows the global distribution, and panel (B) shows England, United Kingdom - the geographical source of the majority of responses $(n=993)$.

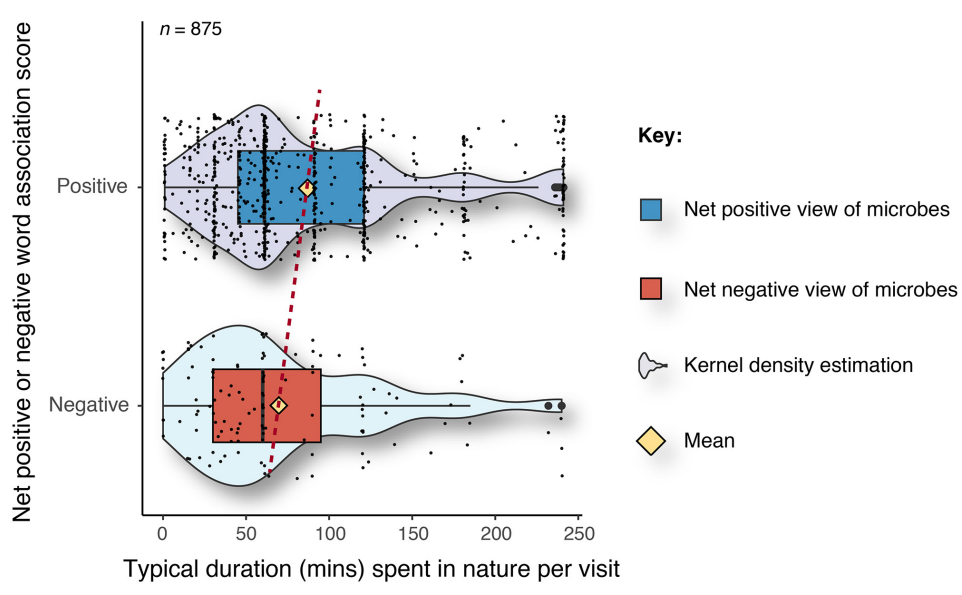

FIGURE 2 | Typical duration spent in natural environments per visit for respondents with net positive and net negative word-association scores. The yellow diamond represents the mean value. The dashed red line is a visual aid to track the difference in means.

(Liddicoat et al., 2019; Robinson et al., 2020). On the other hand, a greater habituation to these kinds of environments and an affinity for natural environments with its diverse life-forms could conceivably reduce one's aversion to microbes in general (as shown with "macro" organisms). It is important to acknowledge here that spending time in natural environments exposes us to a diverse suite of microbial communities (Robinson et al., 2020; Selway et al., 2020) that are thought to have important beneficial effects on our health (Haahtela, 2019; Renz and Skevaki, 2020).
Therefore, whatever the actual directionality of the proposed relationship is (which requires further research to determine), it is likely to have an important impact on our health and could help to ameliorate the negative consequences of germaphobia (e.g., immune dysfunction) (Rook et al., 2003). In one direction (i.e., contingent on factors related to more positive attitudes toward microbes increasing the likelihood that we will spend more time in nature), we could potentially gain the many benefits associated with nature engagement. These include improvements 


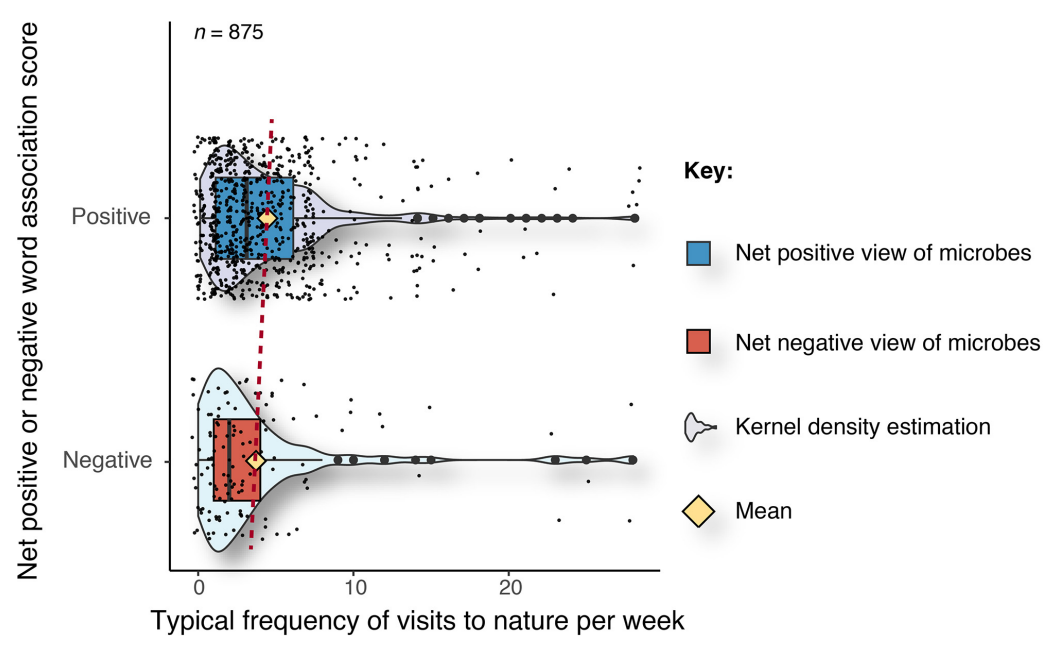

FIGURE 3 | Typical frequency of visits to natural environments per week for respondents with net positive and net negative word-association scores. The yellow diamond represents the mean value. The dashed red line is a visual aid to track the difference in means.

TABLE 1 | Associations between attitudes toward microbes and nature connectedness, adjusting for relative deprivation, education, age and gender.

\begin{tabular}{|c|c|c|c|c|c|}
\hline & Model 1 & Model 2 & Model 3 & Model 4 & Model 5 \\
\hline \multicolumn{6}{|l|}{ Viruses† } \\
\hline Nature connectedness unadjusted & $\begin{array}{c}0.99(0.95,1.02) \\
p=0.54 \text { N.S }\end{array}$ & - & - & - & - \\
\hline Adjusted for IMD§ & - & $\begin{array}{c}0.98(0.89,1.09) \\
p=0.70 \text { N.S }\end{array}$ & - & - & - \\
\hline Adjusted for education level & - & - & $\begin{array}{c}1.07(0.96,1.19) \\
p=0.21 \mathrm{~N} . \mathrm{S}\end{array}$ & - & - \\
\hline Adjusted for age & - & - & - & $\begin{array}{c}0.97(0.90,1.05) \\
p=0.50 \mathrm{~N} . \mathrm{S}\end{array}$ & - \\
\hline Adjusted for gender & - & - & - & - & $\begin{array}{c}1.13(0.85,1.52) \\
p=0.46 \text { N.S }\end{array}$ \\
\hline \multicolumn{6}{|l|}{ All other microbest } \\
\hline Nature connectedness unadjusted & $\begin{array}{c}1.01(0.89,1.16) \\
p=0.86 \mathrm{~N} . \mathrm{S}\end{array}$ & - & - & - & - \\
\hline Adjusted for IMD§ & - & $\begin{array}{c}0.98(0.89,1.09) \\
p=0.70 \text { N.S }\end{array}$ & - & - & - \\
\hline Adjusted for education level & - & - & $\begin{array}{c}1.19(0.75,1.88) \\
p=0.46 \text { N.S }\end{array}$ & - & - \\
\hline Adjusted for age & - & - & - & $\begin{array}{c}1.29(0.94,1.79) \\
p=0.12 \mathrm{~N} . \mathrm{S}\end{array}$ & - \\
\hline Adjusted for gender & - & - & - & - & $\begin{array}{c}0.55(0.17,1.75) \\
p=0.60 \mathrm{~N} . \mathrm{S}\end{array}$ \\
\hline
\end{tabular}

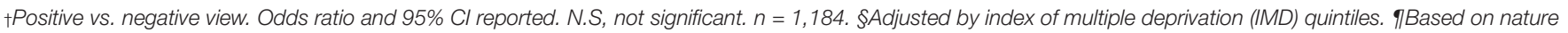
relatedness-6 scale (NR-6).

in immune health (Li, 2010; Rook, 2013), mental health (Birch et al., 2020; Callaghan et al., 2020), and cardiovascular health (Yeager et al., 2020). In the alternative direction (i.e., spending more time in natural environments which may help to establish more positive attitudes toward microbes), we can hypothesize that our positive attitudes toward microbes could conceivably reduce the likelihood that we carry out mass (non-targeted) sterilization of our local environments, which could also have important implications for our health (Jun et al., 2018; Parks et al., 2020; Prescott, 2020; Renz and Skevaki, 2020). This hypothesis requires further research and would benefit from the collection of data on people's actions (e.g., related to environmental avoidance and sterilization). This relationship could also be nondichotomous (or potentially even a virtuous loop) in the sense that our positive attitudes toward microbes may predispose us to spend more time in nature-an act that may enhance our positive attitudes toward microbes, and the feedback continues. This theoretical relationship warrants further research.

Given that we have shown that nature engagement (duration and frequency in nature) associates with positive attitudes 


\section{Bacteria Viruses Fungi Archaea Micro-algae Protozoa \\ 11) 0.73 0.70 0.81 0.86 0.81 0.82 $p=1.0$ $p=<0.01$ $p=<0.01$ $p=<0.01$ $p=<0.01$ $p=<0.01$}

Mean word-association score for those who did identify the corresponding 0.73 taxonomic group as containing microbes*

Mean word-association score for those who did not identify the corresponding taxonomic group as containing microbes* P-value

*Higher mean scores shown in bold text $n=1184$

FIGURE 4 | Differences in mean microbe word-associated scores for respondents who correctly identified a given taxa as being a microbe compared to those who did not identify the taxa as being a microbe. There were significantly higher (in positivity) word-association scores for respondents who correctly identified that fungi, archaea, micro-algae, and protozoa are microbes compared to those who did not.

toward microbes, it would perhaps be expected that nature connectedness may also associate with positive attitudes toward microbes (our second hypothesis). Studies have shown that people who exhibit higher levels of nature connectedness are more likely to spend time in and engage with natural environments (Capaldi et al., 2014, 2015), and reciprocally, spending time in nature can enhance one's nature connectedness (Nisbet et al., 2019; Chawla, 2020). However, the results of our study show that no significant relationship existed between the nature connectedness of our respondents and their attitudes toward microbes. This could be confounded by other factors, however, age, gender, education and deprivation were controlled for with similar non-significant results. It may simply be that a person's affective, cognitive and experiential connection with nature is not an important factor in predicting one's attitude toward microbes. We can only speculate and say that the invisibility of microbes to the human eye could conceivably negate the affective, cognitive and experiential connection that one may establish with, for example, charismatic fauna or aesthetically-appealing flora. There is a deficit in research on people's emotional and cognitive connection with the invisible constituents of the natural world, and as such, future studies focusing on this relationship would be valuable. It is worthwhile to point out that in contrast to macro-level organisms (e.g., birds and trees), it is only recently-evolutionarily speakingthat humans have been aware of diverse microscopic lifeforms, and only in the past few decades have we been able to comprehensively characterize microbial communities and understand their ecology (Hugenholtz and Tyson, 2008). At this stage, it can only be speculated that this may have an effect on the relationship between nature connectedness and attitudes toward microbes, that is, via a lack of a developed emotional link through sense (e.g., sight, sound, touch)-stimuli interactions over evolutionary timescales. Alternatively, this result could be a facet of the nature connectedness instrument used (the NR-6 Scale). Perhaps a more detailed version of the instrument such as the 17item Connectedness to Nature Scale (CNS) (Mayer and Frantz,
2004) would reveal alternative findings. This warrants further research.

Finally, our study shows a significant relationship between basic level of microbial literacy and attitudes toward microbes, which supports our third hypothesis. Previous work has suggested that environmental knowledge can positively affect attitudes toward nature (Sat Gungor et al., 2018; Choe J. H. et al., 2020), although other research suggests this is not important (Qomariah and Prabawani, 2020). In our study, respondents who correctly identified that lesser publicized (as microbes) organisms - such as algae, fungi, archaea, and protozoa - were microbes, showed higher positivity scores toward microbes. This implies that basic microbial literacy may be an important factor in the formation of a person's attitudes toward microbes, and thus could potentially influence the onset of germaphobia. Determining whether any potential influences on people's attitudes subsequently translates into "germaphilic" or microbeappreciative behaviors, requires further research. Interestingly, mean positive scores toward all microbes were significantly lower for those respondents who identified that viruses were microbes compared to those who did not identify viruses as being microbes. Although further research is needed, one explanation could be that the COVID-19 (virus) pandemic had an effect on people's overall view of microbes. This may be unsurprising given the damage the pandemic has caused and the multi-pronged approach taken to try and eliminate the SARS-CoV-2 virus. However, it could conceivably have negative cascading effects on our health by contributing to broader germaphobia.

Microbes are the foundations of our ecosystems and are essential to the survival of all life on Earth (Cavicchioli et al., 2019). We now have the technology to easily characterize and learn about these diverse invisible communities that continuously surround us, providing essential ecosystem services. Although further research is required to build upon our preliminary findings, it is conceivable that in the future, strategies that aim to enhance positive attitudes toward microbes could include the promotion of nature engagement (spending more time 
and more often in nature), which has several important cobenefits for health and wellbeing (Rook, 2013; Birch et al., 2020). Moreover, perhaps in an educational context, greater emphasis can be placed on microbial literacy moving into the future. With a more nuanced awareness of, and mutuallyadvantageous relationship with these diverse, underappreciated, and indispensable lifeforms, germaphobia-associated attitudes can potentially be reduced, while still maintaining the critically important targeted-hygiene and efforts to control infectious diseases.

\section{Limitations}

Our study has some important limitations. Firstly, the results in the study are correlational. Therefore, strict inferences of causation are not possible. Along similar lines, inferences regarding the directionality of the relationships are also not possible. Non-random sampling methods were used in this study. This means accurate calculations of error and representativeness are not possible. Perhaps one of the most important limitations is that self-reported data collection methods come with inherent biases. For example, responder bias-where participants, either intentionally or by accident, choose an untruthful or inaccurate answer, or where people who consider nature important are over-represented in the study. We acknowledge our attitude assessment was limited, and future studies would benefit from investigating behaviors such as environmental avoidance and sterilization. Further controlled research is required to fully unravel the complexities of the observed relationships. There was also a deficit of samples from outside of England, United Kingdom. The study would have benefited from the inclusion of additional international georeferenced samples to be representative on a wider scale. Temporally-objective natureengagement data that represents scenarios before the COVID-19 pandemic, during the pandemic, and after the pandemic would also bring considerable value.

\section{CONCLUSION}

This study suggests that basic microbial literacy and nature exposure may be important in reducing/preventing germaphobia-associated attitudes. As the rise of immunerelated disorders and mental health conditions have been linked to germaphobia, reduced biodiversity, and non-targeted sterilization, our findings point to a simple strategy to potentially help ameliorate these negative consequences, although further research is required to explore this in greater detail. Indeed, a greater emphasis on microbial literacy and promoting time spent in nature could potentially be useful in promoting resilience in human health and more positive/constructive attitudes toward the foundations of our ecosystems - the microorganisms.

\section{REFERENCES}

Arleevskaya, M. I., Aminov, R., Brooks, W. H., Manukyan, G., and Renaudineau, Y. (2019). Shaping of human immune system and metabolic processes by viruses and microorganisms. Front. Microbiol. 10:816. doi: 10.3389/fmicb.2019.00816

\section{DATA AVAILABILITY STATEMENT}

The datasets presented in this study can be found in online repositories. The names of the repository/repositories and accession number(s) can be found below: United Kingdom Data Service ReShare: https://reshare.ukdataservice.ac.uk/ Data Collection: 854604.

\section{ETHICS STATEMENT}

The studies involving human participants were reviewed and approved by University of Sheffield; Department of Landscape Architecture Review Committee. The patients/participants provided their written informed consent to participate in this study.

\section{AUTHOR CONTRIBUTIONS}

JR, RC, and AJ contributed to the conception and design of the study and manuscript internal critical review process and revisions. JR conducted the data analysis, wrote the manuscript, produced the figures, and data visualizations. All authors read and approved the submitted version.

\section{FUNDING}

JR is undertaking a Ph.D. through the White Rose Doctoral Training Partnership (WRDTP), funded by the Economic and Social Research Council (ESRC). Grant code: ES/J500215/1.

\section{ACKNOWLEDGMENTS}

We would like to thank Martin Breed, Chris Bedwell, Vicky Peace, Rachael White, and Kate Robinson for helping to pilot test the questionnaires and for providing feedback on the design.

\section{SUPPLEMENTARY MATERIAL}

The Supplementary Material for this article can be found online at: https://www.frontiersin.org/articles/10.3389/fpsyg. 2021.678752/full\#supplementary-material

Supplementary Appendix A | Online survey questions.

Balloux, F., and van Dorp, L. (2017). Q\&A: what are pathogens, and what have they done to and for us? BMC Biol. 15:91. doi: 10.1186/s12915-017-0433-z

Birch, J., Rishbeth, C., and Payne, S. R. (2020). Nature doesn't judge you-how urban nature supports young people's mental health and wellbeing in a diverse UK city. Health Place 62:102296. doi: 10.1016/j.healthplace.2020.102296 
Callaghan, A., McCombe, G., Harrold, A., McMeel, C., Mills, G., Moore-Cherry, N., et al. (2020). The impact of green spaces on mental health in urban settings: a scoping review. J. Mental Health 18, 1-5. doi: 10.1080/09638237.2020.1755027

Capaldi, C. A., Dopko, R. L., and Zelenski, J. M. (2014). The relationship between nature connectedness and happiness: a meta-analysis. Front. Psychol. 5:976. doi: 10.3389/fpsyg.2014.00976

Capaldi, C. A., Passmore, H. A., Nisbet, E. K., Zelenski, J. M., and Dopko, R. L. (2015). Flourishing in nature: a review of the benefits of connecting with nature and its application as a wellbeing intervention. Int. J. Wellbeing 5, 1-16. doi: 10.5502/ijw.v5i4.449

Cavicchioli, R., Ripple, W. J., Timmis, K. N., Azam, F., Bakken, L. R., Baylis, M., et al. (2019). Scientists' warning to humanity: microorganisms and climate change. Nat. Rev. Microbiol. 17, 569-586.

Chawla, L. (2020). Childhood nature connection and constructive hope: a review of research on connecting with nature and coping with environmental loss. People Nat. 2, 619-642. doi: 10.1002/pan3.10128

Cheung, H., Mazerolle, L., Possingham, H. P., Tam, K. P., and Biggs, D. (2020). A methodological guide for translating study instruments in cross-cultural research: adapting the 'connectedness to nature' scale into Chinese. Methods Ecol. Evol. 11, 1379-1387. doi: 10.1111/2041-210x.13465

Choe, E. Y., Jorgensen, A., and Sheffield, D. (2020). Does a natural environment enhance the effectiveness of Mindfulness-Based Stress Reduction (MBSR)? Examining the mental health and wellbeing, and nature connectedness benefits. Landsc. Urban Plann. 202:103886. doi: 10.1016/j.landurbplan.2020.103886

Choe, J. H., Kim, C. H., and Ri, G. H. (2020). An investigation on the environmental knowledge and attitudes of senior middle school students in the Democratic People's Republic of Korea. Int. Res. Geog. Environ. Educat. 29, 146-162. doi: 10.1080/10382046.2019.1678276

Collado, S., Corraliza, J. A., Staats, H., and Ruiz, M. (2015). Effect of frequency and mode of contact with nature on children's self-reported ecological behaviors. J. Environ. Psychol. 41, 65-73. doi: 10.1016/j.jenvp.2014.11.001

Dannemiller, K. C., Mendell, M. J., Macher, J. M., Kumagai, K., Bradman, A., Holland, N., et al. (2014). Next-generation DNA sequencing reveals that low fungal diversity in house dust is associated with childhood asthma development. Indoor Air 24, 236-247. doi: 10.1111/ina.12072

DeWeerdt, S. (2018). How baby's first microbes could be crucial to future health. Nature 555, S18-S19.

Duron-Ramos, M. F., Collado, S., García-Vázquez, F. I., and Bello-Echeverria, M. (2020). The role of urban/rural environments on Mexican children's connection to nature and pro-environmental behavior. Front. Psychol. 11:514. doi: 10.3389/ fpsyg.2020.00514

Flies, E. J., Clarke, L. J., Brook, B. W., and Jones, P. (2020). Urbanisation reduces the abundance and diversity of airborne microbes-but what does that mean for our health? A systematic review. Sci. Total Environ. 738:140337. doi: 10.1016/j. scitotenv.2020.140337

Gensollen, T., Iyer, S. S., Kasper, D. L., and Blumberg, R. S. (2016). How colonization by microbiota in early life shapes the immune system. Science 352, 539-544. doi: 10.1126/science.aad9378

Guerra, C. A., Heintz-Buschart, A., Sikorski, J., Chatzinotas, A., Guerrero-Ramírez, N., Cesarz, S., et al. (2020). Blind spots in global soil biodiversity and ecosystem function research. Nat. Comm. 11:3870.

Haahtela, T. (2019). A biodiversity hypothesis. Allergy 74, 1445-1456.

Hosaka, T., Sugimoto, K., and Numata, S. (2017). Childhood experience of nature influences the willingness to coexist with biodiversity in cities. Palgrave Commun. 3:17071.

Hugenholtz, P., and Tyson, G. W. (2008). Metagenomics. Nature 455, 481-483.

Jun, S., Drall, K., Matenchuk, B., McLean, C., Nielsen, C., Obiakor, C. V., et al. (2018). Sanitization of early life and microbial dysbiosis. Challenges 9:43. doi: 10.3390/challe9020043

Kettner, H., Gandy, S., Haijen, E. C., and Carhart-Harris, R. L. (2019). From egoism to ecoism: psychedelics increase nature relatedness in a state-mediated and context-dependent manner. Int. J. Environ. Res. Public Health 16:5147. doi: 10.3390/ijerph16245147

Li, M., Fang, A., Yu, X., Zhang, K., He, Z., Wang, C., et al. (2020). Microbiallydriven sulfur cycling microbial communities in different mangrove sediments. Chemosphere 13:128597. doi: 10.1016/j.chemosphere.2020.128597

Li, Q. (2010). Effect of forest bathing trips on human immune function. Environ. Health Prevent. Med. 15, 9-17. doi: 10.1007/s12199-008-0068-3
Liddicoat, C., Sydnor, H., Cando-Dumancela, C., Dresken, R., Liu, J., Gellie, N. J., et al. (2019). Naturally-diverse airborne environmental microbial exposures modulate the gut microbiome and may provide anxiolytic benefits in mice. Sci. Total Environ. 701:134684. doi: 10.1016/j.scitotenv.2019.134684

Mayer, F. S., and Frantz, C. M. (2004). The connectedness to nature scale: a measure of individuals' feeling in community with nature. J. Environ. Psychol. 24, 503-515. doi: 10.1016/j.jenvp.2004.10.001

Nisbet, E. K., and Zelenski, J. M. (2013). The NR-6: a new brief measure of nature relatedness. Front. Psychol. 4:813. doi: 10.3389/fpsyg.2013.00813

Nisbet, E. K., Zelenski, J. M., and Grandpierre, Z. (2019). Mindfulness in nature enhances connectedness and mood. Ecopsychology 11, 81-91. doi: 10.1089/eco. 2018.0061

Obery, A., and Bangert, A. (2017). Exploring the influence of nature relatedness and perceived science knowledge on proenvironmental behavior. Educat. Sci. 7:17. doi: 10.3390/educsci7010017

Parks, J., McCandless, L., Dharma, C., Brook, J., Turvey, S. E., Mandhane, P., et al. (2020). Association of use of cleaning products with respiratory health in a Canadian birth cohort. CMAJ 192, E154-E161.

Prescott, S. L. A. (2020). Butterfly Flaps its Wings: extinction of biological experience and the origins of allergy. Ann Allergy Asthma Immunol. 125, 528-534. doi: 10.1016/j.anai.2020.05.025

Qadir, M. I., and Yameen, I. A. (2019). Questionnaire based study about association between blood oxygen level and Mysophobia. Biomed. J. Sci. Tech. Res. 14, $1-3$.

Qomariah, A., and Prabawani, B. (2020). “The effects of environmental knowledge, environmental concern, and green brand image on green purchase intention with perceived product price and quality as the moderating variable," in Proceedings of the IOP Conference Series: Earth and Environmental Science, Vol. 448, (Bristol: IOP Publishing), 012115. doi: 10.1088/1755-1315/448/1/01 2115

Renz, H., and Skevaki, C. (2020). Early life microbial exposures and allergy risks: opportunities for prevention. Nat. Rev. Immunol. 21, 177-191. doi: 10.1038/ s41577-020-00420-y

Richardson, M., Passmore, H. A., Barbett, L., Lumber, R., Thomas, R., and Hunt, A. (2020). The green care code: how nature connectedness and simple activities help explain pro-nature conservation behaviours. People Nat. 2, 821-839. doi: 10.1002/pan3.10117

Robinson, J. M., and Breed, M. F. (2020). The lovebug effect: is the human biophilic drive influenced by interactions between the host, the environment, and the microbiome? Sci. Total Environ. 28:137626. doi: 10.1016/j.scitotenv. 2020.137626

Robinson, J. M., Cando-Dumancela, C., Liddicoat, C., Weinstein, P., Cameron, R., and Breed, M. F. (2020). Vertical stratification in urban green space aerobiomes. Environ. Health Persp. 128:117008. doi: 10.1289/ehp7807

Rook, G. A. (2013). Regulation of the immune system by biodiversity from the natural environment: an ecosystem service essential to health. Proc. Natl. Acad. Sci. U.S.A. 110, 18360-18367. doi: 10.1073/pnas.1313731110

Rook, G. A., Martinelli, R., and Brunet, L. R. (2003). Innate immune responses to mycobacteria and the downregulation of atopic responses. Curr. Opin. Allergy Clin. Immunol. 3, 337-342. doi: 10.1097/00130832-20031000000003

Roslund, M. I., Puhakka, R., Grönroos, M., Nurminen, N., Oikarinen, S., Gazali, A. M., et al. (2020). Biodiversity intervention enhances immune regulation and health-associated commensal microbiota among daycare children. Sci. Adv. 6:eaba2578. doi: 10.1126/sciadv.aba2578

Sat Gungor, B., Chen, J., Wu, S. R., Zhou, P., and Shirkey, G. (2018). Does plant knowledge within urban forests and parks directly influence visitor pro-environmental behaviors. Forests 9:171. doi: 10.3390/f9040171

Selway, C. A., Mills, J. G., Weinstein, P., Skelly, C., Yadav, S., Lowe, A., et al. (2020). Transfer of environmental microbes to the skin and respiratory tract of humans after urban green space exposure. Environ. Int. 145:106084. doi: 10.1016/j.envint.2020.106084

Smart Survey (2020). Smart Survey Online Surveys. Available online at: https: //www.smartsurvey.co.uk/ (accessed Jaunuary 03, 2020).

Stein, M. M., Hrusch, C. L., Gozdz, J., Igartua, C., Pivniouk, V., Murray, S. E., et al. (2016). Innate immunity and asthma risk in Amish and Hutterite farm children. N. Engl. J. Med. 375, 411-421. doi: 10.1056/nejmoa150 8749 
Timmis, K., Cavicchioli, R., Garcia, J. L., Nogales, B., Chavarría, M., Stein, L., et al. (2019). The urgent need for microbiology literacy in society. Environ. Microbiol. $21,1513-1528$.

Trivedi, P., Leach, J. E., Tringe, S. G., Sa, T., and Singh, B. K. (2020). Plantmicrobiome interactions: from community assembly to plant health. Nat. Rev. Microbiol. 18, 607-621. doi: 10.1038/s41579-020-0412-1

Whitburn, J., Linklater, W., and Abrahamse, W. (2020). Meta-analysis of human connection to nature and proenvironmental behavior. Conserv. Biol. 34, 180193. doi: $10.1111 /$ cobi.13381

Yeager, R. A., Smith, T. R., and Bhatnagar, A. (2020). Green environments and cardiovascular health. Trends Cardiol. Med. 30, 241-246. doi: 10.1016/j.tcm. 2019.06.005

Yeoh, Y. K., Zuo, T., Lui, G. C., Zhang, F., Liu, Q., Li, A. Y., et al. (2021). Gut microbiota composition reflects disease severity and dysfunctional immune responses in patients with COVID-19. Gut 70, 698-706. doi: 10.1136/gutjnl2020-323020
Zemke, D. M., Neal, J., Shoemaker, S., and Kirsch, K. (2015). Hotel cleanliness: will guests pay for enhanced disinfection? Int. J. Contemp. Hospit. Manag. 27, 690-710. doi: 10.1108/ijchm-01-2014-0020

Zobell, C. E., and Rittenberg, S. C. (2011). Microbiology by numbers. Nat. Rev. Microbiol. 9:628. doi: 10.1038/nrmicro2644

Conflict of Interest: The authors declare that the research was conducted in the absence of any commercial or financial relationships that could be construed as a potential conflict of interest.

Copyright $\odot 2021$ Robinson, Cameron and Jorgensen. This is an open-access article distributed under the terms of the Creative Commons Attribution License (CC BY). The use, distribution or reproduction in other forums is permitted, provided the original author(s) and the copyright owner(s) are credited and that the original publication in this journal is cited, in accordance with accepted academic practice. No use, distribution or reproduction is permitted which does not comply with these terms. 